\title{
Drug Utilization Study in Patients Admitted in Medical Wards in a Tertiary Care Rural Hospital in North Kashmir
}

\author{
Rubeena Maqbool' ${ }^{1}$, Shakil U. Rehman ${ }^{2}$ \\ ${ }^{1}$ Department of Pharmacology, GMC, Baramulla, Jammu and Kashmir, India. \\ ${ }^{2}$ Department of Pharmacology, GMC, Baramulla, Jammu and Kashmir, India.
}

\section{ABSTRACT}

\section{BACKGROUND}

Drug utilization studies are an integral part of medical audit and are undertaken to monitor and evaluate prescribing practices. Drug utilization studies are rarely conducted in rural settings where regulations are weak, and expertise and manpower are low.

\section{METHODS}

This prospective observational study was conducted at Govt. Medical College, Baramulla, by reviewing the inpatient records of patients admitted in the medical wards and staying in the hospital for at least one night. Data is presented as percentages.

\section{RESULTS}

A total of 300 patients were enrolled and 260 were included for the study, out of which $43.07 \%$ were male and $56.92 \%$ were females. $50 \%$ of patients were in age group of 18 to 45 years while as $25 \%$ of the patients belonged to age group 46 to 65 years and > 65 years. The diagnosis of GI disorders comprised of 45\%; respiratory, renal, endocrine and hepato-biliary disease was 24.25\%, 6.92\%, $23 \%$ and $5 \%$ respectively. $5.30 \%$ of patients were having fever only, $3.46 \%$ were of miscellaneous group while $2.30 \%$ were having organophosphorous poisoning. The drugs most often prescribed were antimicrobials $88.46 \%$, analgesics $55 \%$, and GI drugs $70 \%$. Among the antimicrobials, $63.47 \%$ were cephalosporins, $26.95 \%$ quinolones, $5.21 \%$ antiprotozoal, $17.39 \%$ cephalosporins and antiprotozoals. $39.16 \%$ analgesics were NSAIDs; opioids comprised $18.88 \%$ and antispasmodics $40.55 \%$. The GIT drugs comprised $7.69 \%$ antiemetic, $78.02 \%$ PPIs $9.89 \%$ antispasmodic combination of anti-emetics, and $4.39 \%$ PPIs. Other drugs were $66.15 \%$ of which $95.34 \%$ were IV fluids. $85.77 \%$ were discharged while as $3.46 \%$ were referred and $3.07 \%$ had LAMA. ADRs were seen in $6.15 \%$ of patients. Antibiotics were started empirically without culture sensitivity tests being done.

\section{CONCLUSIONS}

The present study highlights the problem of over-prescription of antimicrobials. The need of the hour is to formulate guidelines for hospital antibiotic usage.

\section{KEY WORDS}

Drug Utilization, Rational Use, Antimicrobials, Drugs
Corresponding Author:

Dr. Shakil U. Rehman,

Department of Pharmacology,

GMC, Baramulla,

Jammu and Kashmir, India.

E-mail:drshakil924@gmail.com

DOI: $10.14260 /$ jemds/2020/310

Financial or Other Competing Interests: None.

How to Cite This Article:

Maqbool R, Rehman SU. Drug utilization study in patients admitted in medical wards in a tertiary care rural hospital in North Kashmir. J. Evolution Med. Dent. Sci. 2020;9(17):1423-1426, $10.14260 /$ jemds $/ 2020 / 310$

Submission 26-02-2020, Peer Review 14-04-2020, Acceptance 22-04-2020, Published 27-04-2020.

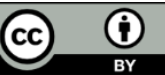




\section{BACKGROUND}

Drug utilization studies are integral part of medical audit and they are often undertaken to monitor and evaluate prescribing practices. The aim of drug utilization study is to promote rational and appropriate use of drugs at lowest possible dose and cost. ${ }^{1}$ Rational use of medicine requires that patients receive medication appropriate to their clinical needs, in doses that meet their individual requirements for an adequate period of time and at the lowest cost to them and their community (WHO 1985). Irrational medicine use occurs with poly pharmacy or with underuse or incorrect use of effective medicines. Irrational medicine use negatively affects the quality of medicine therapy, increase health care cost, may cause adverse drug reactions, emergence of drug resistance there by hamper the health care delivery services.

The most commonly seen irrational use of medicines is excessive use of antibiotics. ${ }^{2}$ the rise in the consumption of antibiotics results in further increase in resistance to antibiotics. Existing evidence suggests that there is a causal association between antimicrobial usage in hospital and antimicrobial resistance. ${ }^{3}$ Chance of development of resistant strain to antibiotics if misused will lead to unnecessary adverse effects and drug interaction. So, information about antibiotic use patterns is necessary for a constructive approach to problems that arise from the use of multiple antibiotics available.

With 20, 00,000 people losing battle to antimicrobial resistance per year and another 10 million projected to die from it by 2050; antimicrobial resistance (AMR) is killing more people than cancer and road traffic accidents combined together. ${ }^{4}$ It is not needless to mention here that, India ranks first among all countries of the world in total consumption of antibiotics for human use. ${ }^{5}$ A total of $12.9 \times 10^{9}$ units of antibiotics with 10.7 units per individual were consumed in India in 2010 alone. ${ }^{6}$ These figures, though threatening, are not surprising for India where antibiotics are used day in and day out.7,8

Thus antibiotic resistance is now a major threat to public health ${ }^{9}$ Besides India has been referred to as "the AMR capital of the world," 10 hence a need to undertake a drug utilization study which is an approved, systematic process that captures, reviews, analyses, and interprets aggregate medication use data within specific health care environments. ${ }^{11}$

These studies are helpful to identify the prescribing trends and may lead to interventions to enhance prescribing behaviour. The study of prescribing patterns seeks to monitor, evaluate and suggest modifications in practitioners prescribing habits so as to make medical care rational and cost effective. ${ }^{12}$ Very few studies are available nationally and internationally which have observed prescribing patterns among medical practitioners in patients admitted in medical ward in a Tertiary Care hospital among indoor patients of general medicine. ${ }^{13}$

Hence this study was conducted to evaluate the rationality of prescriptions with a special emphasis to evaluate the antibiotic prescribing patterns and analyse the chances of antimicrobial resistance.

\section{METHODS}

The prospective observational study was conducted at Government Medical College Baramulla, which is a newly established 300 bedded rural tertiary health care facility, after seeking permission from concerned Ethical Committee of the institution. All patients admitted in the medical wards from July to September 2019 and who stayed in the hospital for at least one night during the study period were included in the study. The patients who were less than 18 years of age, critically ill patients and patients not ready to give consent for the study were excluded from the study. The prescriptions were evaluated on the first day of the admission and data was entered in tables and diagrams.

\section{Variables}

Patient information was analysed for age, sex, diagnosis received, drugs prescribed, antibiotic treatment, IV fluids and results presented as percentages.

\section{RESULTS}

Total patients enrolled were 300. After subjecting these enrolled patients to screening, and fulfilling the inclusion and exclusion criteria, patients included in study were 260 . Out of the 260 patients, $43.07 \%$ were male while as females comprised of $56.92 \% .50 \%$ of patients were in age group of 18 to 45 years while as $25 \%$ of the patients belonged to age group 46 to 65 years and $>65$ years of age respectively (table 1). The drugs prescribed were $88.46 \%$ of antimicrobials, GI drugs $70 \%$, analgesics 55\%, other drugs $66.15 \%$ (table 2). Among the antimicrobials, $63.47 \%$ were cephalosporins, quinolones $26.95 \%$, antiprotozoal $5.21 \%$ cephalosporins and antiprotozoal 1.73\% (table 3). 39.46\% of analgesics were NSAID; opioids comprised $18.88 \%$ and antispasmodics $40.55 \%$ (table 4 ).

\begin{tabular}{|c|c|c|c|}
\hline \multirow{2}{*}{ Age } & \multicolumn{2}{|c|}{ Sex } & \multirow{2}{*}{ Total n $(\%$} \\
\hline & Male n (\%) & Female n (\%) & \\
\hline $18-45$ yrs. & 43 & 87 & $130(50 \%)$ \\
\hline $45-65$ yrs. & 34 & 31 & $65(25 \%)$ \\
\hline$>65$ yrs. & 35 & 30 & $65(25 \%)$ \\
\hline Total n (\%) & $112(43.07 \%)$ & $148(56.92 \%)$ & 260 \\
\hline \multicolumn{4}{|c|}{ Table 1. Age and Sex Distribution of the Study Population $(n=260$} \\
\hline
\end{tabular}

\begin{tabular}{|ccc|}
\hline Drug & $\mathbf{n = 2 6 0}$ & Percentage \\
Antimicrobials & 230 & $88.46 \%$ \\
Analgesics & 143 & $55 \%$ \\
GI drugs & 182 & $70 \%$ \\
Other drugs & 172 & $66.15 \%$ \\
\hline \multicolumn{3}{|c}{ Table 2. Drugs Prescribed } \\
\hline
\end{tabular}

\begin{tabular}{|ccc|}
\hline Antimicrobial & n=230 & Percentage \\
Cephalosporins & 146 & $63.47 \%$ \\
Quinolones & 62 & $26.95 \%$ \\
Penicillin & 4 & $1.73 \%$ \\
Antiprotozoals & 12 & $5.21 \%$ \\
Cephalosporin and antiprotozoal & 4 & $1.73 \%$ \\
Cephalosporin and quinolones & 2 & $0.86 \%$ \\
\hline \multicolumn{2}{|c|}{ Table 3. Antimicrobials Prescribed } \\
\hline
\end{tabular}

\begin{tabular}{|ccc|}
\hline Analgesic & n=143 & Percentage \\
NSAID & 56 & $39.46 \%$ \\
Opioids & 27 & $18.88 \%$ \\
Antispasmodic & 58 & $40.55 \%$ \\
\hline \multicolumn{2}{|c|}{ Table 4. Analgesic Drugs Prescribed } \\
\hline
\end{tabular}




\begin{tabular}{|ccc|}
\hline GIT Drugs & $\mathbf{n = 1 8 2}$ & Percentage \\
Antiemetic & 14 & $7.69 \%$ \\
PPIs & 142 & $78.02 \%$ \\
Antispasmodics & 18 & $9.89 \%$ \\
Antiemetic and PPIs & 8 & $4.39 \%$ \\
\hline \multicolumn{3}{|c}{ Table 5. GI Drugs Prescribed } \\
\hline \multicolumn{3}{c}{}
\end{tabular}

The GIT drugs comprised 7.69\% antiemetic, PPIs 78.02\%. Antispasmodic 9.89\% combination of antiemetic, and PPIs $4.39 \%$ (table 5 ). The other drugs comprised $66.15 \%$ of which IV fluids were $95.34 \%$. $85.77 \%$ were discharged while as $3.46 \%$ were referred and $3.07 \%$ had LAMA. Adverse drug reactions were seen in $6.15 \%$ of patients. Antibiotic were started empirically without culture sensitivity being done.

\section{DISCUSSION}

Appropriate drug utilization studies are needed for evaluating proper utilization of drugs for efficacy, safety, convenience and economic aspects. ${ }^{14}$ Drug utilization research can provide useful information to health care providers \& policy makers. It offers the prospect of improving the quality of pharmacotherapy. In the present study we tried to find out the pattern of drug utilization taking in view rationality of drug prescription with special emphasis on antimicrobial prescription. The association between antibiotic use and the emergence of antibiotic resistance is well-established. ${ }^{15}$ Resistance to antibiotics is considered a global health care crisis which currently threatens the advances of modern medicine. ${ }^{16}$ Increased and inappropriate antibiotic use among outpatients and in hospitals has been noted worldwide ${ }^{17}$ Antimicrobial stewardship (AMS) programmes aim to optimize the quality of antibiotic prescribing while minimizing levels of antimicrobial resistance. ${ }^{18}$

In the present study the females outnumber males, out of the 260 patients, $43.07 \%$ were male while as females comprised of $56.92 \%$. In case of females in the present study because of occupational, mental stress or other social factors might have led to increased drug utilization with the result causing further increase in morbidity. The age group involved is young and economically productive group which therefore points to the role of counsellors in imparting proper guidelines regarding the health care practices. Age and gender distribution reported by Meher et $\mathrm{al}^{19}$ and Choudhary et al. ${ }^{20}$ where in $62 \%$ were male and $38 \%$ were female, with age range between $15-90$ years is different from that observed in the present study.

The main highlighting point in the study is the use of antimicrobial agents, the drugs prescribed were $88.46 \%$ of antimicrobials, that too the cephalosporins. Among the antimicrobials, $63.47 \%$ were cephalosporins. The antimicrobial agent was used empirically and by parenteral routes. High use of antimicrobials in the present study can be compared to an observation by Bhansali et $\mathrm{al}^{21}$ where majority of the patients in surgery department of a hospital in western India were given antibiotics unnecessarily. These findings were also comparable to the similar findings in the study conducted in Western Nepal by Shankar et al. ${ }^{22}$

Majority of the patients in our study were on parenteral antibiotics. It is worthwhile to mention here that culture should be taken before treatment when indicated and treatment potentially changed based on susceptibility report.

The next drug which is prescribed in greater percentage after antibiotic are PPIs followed by other gastro protective drugs. The overuse of drugs for GI disorders is indicative of the chances of greater incidence of involvement of GI disorders hence the need for proper counselling and education about proper dietary practices but the overuse of PPIs needs to be justified by the prescribers in view of Rational drug prescribing. Among the other drugs IV fluids were being used in greater percentage of patients. The next noteworthy feature of concern is the use of antispasmodic among analgesics which is to be justified in the light of rational drug prescribing by the concerned prescribers. Here the emphasis should be laid on that the prescriber should clearly adhere to the five rights of drug administration. ${ }^{23}$

\section{Limitations and Recommendations}

Since the study was conducted in the medicine indoor wards only, so the overall generalized pattern of drug utilization was not reflected. Less number of patients were used in the present study hence multicentric studies are needed to substantiate the present data. So, the recommendations of the study are formulation and strict implementation of standard treatment guidelines in the hospitals regarding antibiotics and use of culture sensitivity and rational use of medicines in general.

\section{CONCLUSIONS}

The present study highlights the problem of overprescription of antimicrobials and excessive use of proton pump inhibitors. The overuse of proton pump inhibitors indicative of increase in GI related disorders may be dealt with the inclusion of proper counselling at primary health care so that preventive measures are incorporated at grassroot level. Appropriate antimicrobial stewardship programmes should also be incorporated not only to limit the use of inappropriate agents but implementation of guidelines for hospital antibiotic usage should also be prioritized in order to encourage the appropriate selection, dosing and duration of antimicrobial therapy among the prescribers so as to achieve optimal efficacy in managing infections and standardizing the use of antimicrobial therapy. Finally, the need of the hour is to lay stress on conducting of educational programs to prescribers besides teaching clinical pharmacology and rational use of drugs to undergraduate students.

\section{REFERENCES}

[1] Shalini S, Ravichandran V, Mohanty BK, et al. Drug utilization studies - an overview. Intern J Pharma Sci Nanotech 2010;3(1):803-10.

[2] Ebrahimzadeh MA, Shokrzadeh M, Ramezani A. Utilization pattern of antibiotic in different wards of specialized Sari Emam University Hospital in Iran. Pak J Biol Sci 2008;11(2):275-9. 
[3] Rogues AM, Placet-Thomazeau B, Parneix P, et al. Use of antibiotics in hospitals in south western France. J Hospital Infect 2004;58(3):187-92.

[4] O'Neill J. Tackling drug-resistant infections globally: final report and recommendations. The review on antimicrobial resistance. 2016. https:amrreview/site/default/files/AMR/

[5] Taneja N, Sharma M. Antimicrobial resistance in the environment: The Indian Sceniorio. Indian J Med Res 2019;149(2):119-28.

[6] Van Boecket TP, Gantra S, Ashok A, et al. Global antibiotic consumption 2000 to 2010. An analysis of national pharmaceutical sales lancet infect dis 2014;14:742-50.

[7] Holloway K, Mathal E, Sorensen I, et al. Communitybased surveillance of antimicrobial use and resistance in resource constrained settings: report on five pilot projects. World Health Organization, Geneva: 2009 (accessed on April 15, 2007). http:apps.Who.int/medicinedocs/documents/s6168e8

[8] Holloway KA, Gandhi A, Patel P, et al. An evaluation of knowledge, attitude and practices about policies to promote inappropriate use: reports from country situational analyses. BMJ 2017;358:2291.

[9] World Health Organization. Fact sheet May 2012. (Assessed on 16/02/2013). http://www.who.int/mediacentre/factsheets /fs365 /en/index.html\#

[10] Chaudary D, Tomar P. Antimicrobial resistance: the next BIG pandemic. Int J Community Med Public Health 2017;4(8):2632-6.

[11] Truter I. A review of drug utilization studies and methodologies. Jordan Journal of Pharmaceutical Sciences 2008;1(2):91-104.

[12] Padwal SL, Motghare VM, Kulkarni MD, et al. Drug utilization in burn patients admitted in wards of a Rural Tertiary Care Teaching Hospital. International Journal of Pharmacology and Therapeutics 2013;3(3):19-27.

[13] Meena VK, Atray M, Agrawal A. Evaluation of drug utilization pattern in indoor patients of medicine department at tertiary care teaching hospital in Southern Rajasthan. Int J Pharm Sci Res 2016;7(9):3835-40.
[14] Leekha S, Terrell CL, Edson RS. General principles of antimicrobial theory. Mayo Clinic Proceeding 2011;86(2):156-67.

[15] Datta S, Wattal C, Goel N, et al. A ten year analysis of multi-drug resistant blood stream infections caused by Escherichia coli and Klebsiella Pneumoniae in a tertiary care hospital. Indian J Med Res 2012;135(6):907-12.

[16] Polk RE, Fox C, Mahoney A, et al. Measurement of adult antibacterial drug use in 130 US hospitals: comparison of defined daily dose and days of therapy. Clin Infect Dis 2007;44(5):664-70.

[17] Goel N, Wattal C, Oberoi JK, et al. Trend analysis of antimicrobial consumption and development of resistance in non-fermenters in a tertiary care hospital in Delhi, India. J Antimicrob Chemother 2011;66(7):162530.

[18] Harmeet SR, Nagarani MA, Moushumi R. A study on the drug prescribing pattern and use of antimicrobial agents at tertiary care teaching hospital in eastern Nepal. Indian J Pharmacol 1998;30(5):175-80.

[19] Meher BR, Mukharjee D, Udayshankar. A study on antibiotic utilization pattern in a general medicine ward of a tertiary care teaching hospital. Journal of Chem and Pharma Res 2014;6(7):1847-9.

[20] Chaudhary PK, Maurya AK, Jain A, et al. Drug utilization pattern in medicine department in a tertiary care teaching hospital in Uttar Pradesh. Indo Am Journal of Pharmaceutical Research 2015.

[21] Bhansali NB, Gosai TR, Dholaria NK, et al. Drug utilization study in post-operative patients in surgical ward of a tertiary hospital attached with medical college. Der Pharmacia Lettre 2013;5(1):251-7.

[22] Shankar RP, Partha P, Shenoy NK, et al. Prescribing patterns of antibiotics and sensitivity patterns of common micro-organisms in the Internal Medicine ward of a tertiary hospital in Western Nepal: a prospective study. Ann of Clinical Microbiol and Antimicrobials 2003;2:7.

[23] Grissinger M. The five rights: a destination without a map. Pharmacy and Therapeutics 2010;35(10):542. 